\title{
The validity of O'Sullivan's classification system (CS) for a sub-group of NS-CLBP with motor control impairment (MCI): Overview of a series of studies and review of the literature
}

\author{
Wim Dankaerts ${ }^{\mathrm{a}, \mathrm{b}, *}$, Peter O'Sullivan ${ }^{\mathrm{c}}$ \\ ${ }^{\text {a }}$ Catholic University, Leuven, Belgium \\ ${ }^{\mathrm{b}}$ University College Limburg, AUHL-PHL, Hasselt, Belgium \\ ${ }^{\mathrm{c}}$ Curtin University of Technology, Perth, Australia
}

\section{A R T I C L E I N F O}

\section{Article history:}

Received 10 May 2010

Received in revised form

7 October 2010

Accepted 13 October 2010

\section{Keywords:}

Chronic low back pain

Motor control

Classification

\begin{abstract}
A B S T R A C T
Chronic Low Back Pain (LBP) remains a common, recalcitrant and costly problem for the individual sufferer and for society. Effective treatments that reduce the social and economic burden have yet to be established for the majority of chronic LBP cases. Lack of evidence for specific interventions has been blamed on the heterogeneity of the chronic LBP population as well as a lack of a patient centred biopsycho-social approach. This issue of heterogeneity has resulted in classification being considered the highest research priority in the area of chronic LBP. The potential for a 'wash-out effect' caused by the heterogeneity of the chronic LBP populations sampled for randomised controlled clinical trials (RCTs), has driven the need for classifying patients with nonspecific chronic LBP. A summary of a series of studies is outlined in this review paper. They represent a comprehensive investigation into the validity of O'Sullivan's proposed mechanism-based classification system (CS) for a sub-group of localized mechanically provoked nonspecific chronic LBP with motor control impairment (MCI). Further, the findings of these studies are discussed in relation to the relevant literature and the clinical implications arising are presented. Finally, the limitations of this research are outlined and recommendations for future research are made.
\end{abstract}

(c) 2010 Elsevier Ltd. All rights reserved.

\section{O'Sullivan's classification system (CS) for a sub-group of nonspecific chronic LBP with motor control impairment (MCI)}

Chronic lumbo-pelvic pain disorders are complex and multidimensional in nature. It is well acknowledged that these disorders can be associated with changes in physical, neurophysiology, lifestyle, altered motor control, psycho-social factors and in a small number of cases patho-anatomical factors (Waddell, 2004). There is a considerable debate as to the significance of these different factors and what is cause and effect. The majority of lumbo-pelvic pain disorders have no diagnosis leaving a management vacuum. From this perspective, classification of nonspecific chronic low back pain (LBP) disorders into sub-groups, based on the mechanism(s) underlying the disorder, is considered critical to ensure appropriate management.

\footnotetext{
* Corresponding author. Musculoskeletal Research Unit, Department of Rehabilitation Sciences, Faculty of Kinesiology and Rehabilitation Sciences, University of Leuven, Tervuursevest 101, B-3001 Leuven, Belgium. Tel.: +32 163 29070; fax: +32 16329197.

E-mail address: wim.dankaerts@faber.kuleuven.be (W. Dankaerts).
}

In order to identify and sub-classify specific sub-groups of nonspecific chronic LBP patients within a mechanism-based CS, O'Sullivan proposed a division between disorders of the central nervous system (such as regional pain disorders, neuropathic pain and fibromyalgia), and localized pain disorders that have a peripheral pain pattern and mechanical pain behaviour (O'Sullivan, 2005). Similar divisions have previously been proposed by experts in the pain field (Bogduk and Merskey, 1994; Latremoliere and Woolf, 2009). It was further proposed that this second group can be classified into a large group of patients with localized nonspecific chronic LBP, with either movement impairments or control impairments that act as a peripheral driver of their disorder. Pain disorders associated with a 'movement impairment' classification are usually characterized by avoidant pain behaviour and are associated with a loss of normal physiological lumbo-pelvic mobility in the direction of pain provocation.

Those with a 'MCl' classification, sub-group patients with localized nonspecific chronic LBP based on pain behaviours related to adopting provocative spinal postures and movement patterns (O'Sullivan, 2004, 2005), where there is no movement impairment in the direction of pain provocation. Five distinct (direction based) 
patterns of MCI have been described in detail (O'Sullivan, 2004, 2005). These sub-groups of $\mathrm{MCI}$ consist of the; flexion pattern (FP), active extension pattern (AEP), passive extension pattern (sagittal plane), lateral shifting pattern (frontal plane) and a multidirectional pattern (combination of the others).

O'Sullivan's CS is based on a process of diagnostics (Elvey and O'Sullivan, 2004) to make a clinical determination as to whether the patient has a classification of $\mathrm{MCI}$ (maladaptive movement behaviours) or whether the altered movement behaviour is simply a secondary effect of another process (adaptive movement behaviours). 'Diagnostics' places a strong emphasis on the correlation between the subjective history, radiology, pain behaviour, lifestyle factors, physical examination findings (examining the articular, neural and motor control systems) and screens for serious pathology ('red flags') and dominant psycho-social factors (including negative beliefs, coping, fear avoidance beliefs, stress, anxiety, depression, catastrophising etc). As a result of this diagnostic process the relationship between the patient's pain and the $\mathrm{MCI}$ is determined (Elvey and O'Sullivan, 2004; O'Sullivan, 2005). Where it is determined that the $\mathrm{MCI}$ is directly related to the provocation of the pain disorder, a classification of $\mathrm{MCI}$ with respect to the direction of pain provocation is made. Within this system there is the potential for peripheral and central pain drivers to co-exist, where a patient may have an MCI with associated cognitive factors such as fear avoidance behaviours and poor coping strategies reinforcing the disability associated with the disorder (O'Sullivan, 2005). Rather than a reductionist approach to decision making based on a series of prediction rules (Fritz et al., 2003), this approach provides a broad framework for the identification of underlying mechanisms and behaviours associated with a pain disorder in order to guide management.

\section{A summary of the findings of a series of five studies on the validation of a proposed CS for nonspecific chronic LBP}

Following the recommendations emerging from the literature to validate existing CS's (Riddle, 1998; Petersen et al., 1999; Ford, 2002; McCarthy et al., 2004), this review paper provides an accumulation of evidence supporting the validity of a mechanism-based CS for a sub-group of nonspecific chronic LBP with a classification of $\mathrm{MCI}$. A step-wise validation process for the classification of LBP, consisting of accumulating levels of evidence was undertaken. Different methods were developed for each of these stages and were then applied to the CS.

The following section summarizes the findings of five studies conducted by Wim Dankaerts as part of his doctoral research at Curtin University (referred to as Studies I-V).

\section{Can clinicians agree on the classification of nonspecific chronic LBP patients with MCI? (Study I)}

The results of Study I (Dankaerts et al., 2006c) revealed that there was almost perfect agreement (kappa-coefficient 0.96; \%-ofagreement 97\%) between two 'expert' clinicians, in identifying and classifying patients with nonspecific chronic LBP into five specific sub-groups of $\mathrm{MCI}$ based on a comprehensive clinical examination. Study I also demonstrated substantial agreement (mean Kappacoefficients 0.61 and mean \%-of-agreement $70 \%$ ) across all five proposed MCI patterns based on combined subjective case reports and video observation of postures and movements between clinicians (physiotherapists and medical doctors) in two geographically separate regions. It was concluded that clinicians could agree upon the classification of nonspecific chronic LBP patients with MCI supporting its reliability. The fact that clinicians agree supports the clinical viability and face validity.
In support of this work a recent study by Fersum et al. (2009a) confirmed a moderate to substantial inter-tester reliability of the CS system for a wider range of patients within the nonspecific chronic LBP population, including patients with $\mathrm{MCI}$. Both the studies (Dankaerts et al., 2006c; Fersum et al., 2009a) demonstrated that familiarity with the CS influenced the reliability results, demonstrating higher agreement among raters with more CS training $(<100 \mathrm{~h})$.

\section{Can laboratory tests evaluating posture in sitting discriminate two sub-groups of nonspecific chronic LBP (AEP and FP) from no-LBP? Is classification into sub-groups important for clinical research in subjects who report aggravation of LBP in sitting? (Study II)}

While there is consensus in the literature that sitting commonly exacerbates and perpetuates LBP, there is clearly a paucity of studies that had quantitatively examined different sitting postures among the LBP population (Dankaerts et al., 2006b). In Study II lumbo-sacral posture was measured during "usual" and "slumped" sitting in 33 nonspecific chronic LBP patients and 34 asymptomatic subjects using an electromagnetic measurement device. Before testing nonspecific chronic LBP patients were sub-classified by two blinded clinicians. Twenty patients were classified with a flexion $\mathrm{MCI}$ and 13 with an AEP MCI (see Table 1 for inclusion criteria, clinical features and exclusion criteria for FP and AEP classification).

The results of Study II (Dankaerts et al., 2006b) showed that, when nonspecific chronic LBP patients were pooled into one group no differences were detected in lumbo-sacral posture during usual sitting when compared to controls. In contrast, analyses based on sub-classification revealed that patients classified with an AEP sat with a more lordotic posture at the symptomatic lower lumbar spine, whereas patients with an FP sat with a more kyphotic posture, when compared to healthy controls. Further, both groups of nonspecific chronic LBP patients had less ability to change their lumbo-sacral posture when asked to slump from usual sitting when compared to controls.

The irony with these patients is that they adopt the very postures that they report they are sensitized to, they have difficulty varying from these positions and appear to have little awareness that they do this. This would appear to be a maladaptive behaviour.

It was concluded that discriminative differences in lumbo-sacral sitting posture existed in these sub-groups that supported the proposed CS. The heterogeneity of the nonspecific chronic LBP subjects' posture when pooled highlights the importance of subclassification in clinical research and provided evidence for construct validity of the CS.

\section{Can laboratory tests evaluating trunk muscle activity in sitting discriminate two clinical sub-groups of nonspecific chronic LBP with a classification of MCI (AEP and FP) from no- LBP? Is classification of sub-groups important for clinical research into muscle activation patterns in subjects who report aggravation of LBP in sitting? (Study III)}

The results of Study III (Dankaerts et al., 2006a) demonstrated that no differences in trunk muscle activity were observed between healthy controls and a pooled nonspecific chronic LBP group in usual sitting. However, when the CS was applied differences were identified. When compared to controls the AEP group presented with high levels of co-contraction of superficial fibres of lumbar multifidus $(+12 \%)$, ilio-costalis lumborum pars thoracis $(+36 \%)$ and transverse fibres of internal oblique $(+43 \%)$. The FP group had less activation of these muscles than the AEP groups and showed a trend towards lower activation when compared to the controls 
Table 1

Inclusion criteria and clinical features and exclusion criteria for flexion pattern and active extension pattern classification.

Inclusion criteria for nonspecific chronic LBP with motor control impairment of flexion pattern or active extension pattern

History of chronic ( $>3$ months) nonspecific (no radiologic diagnosis)

LBP with at least moderate disability (Revised Oswestry score $>15 \%$ )

Pain localized to the lower lumbar spine (L4-L5 or L5-S1) region

with minimal radiation

Absence of red flags

Absence of dominant yellow flags

Clear mechanical basis of disorder: specific postural and functional movements that aggravate and ease symptoms; relief of symptoms

when reducing the strain to the symptomatic spinal segment in the provocation direction

Associated impairments in the control of the motion segment(s)

in the provocative movement direction(s)

Absence of impaired movement of the symptomatic segment in the painful direction of movement or loading (based on clinical joint motion palpation examination)

Clinical diagnosis of a flexion pattern or active extension pattern disorder both clinicians (independently) agreed upon the diagnosis

Key clinical features flexion pattern

Aggravation of symptoms with movements and postures involving

flexion of the lower lumbar spine

Loss of segmental lordosis at symptomatic level, difficulty assuming

and/or maintaining neutral lordotic postures with a tendency to drop into flexion

Pain relief with spinal extension

Key clinical features active extension pattern

Aggravation of symptoms with movements and postures involving

extension of the lower lumbar spine (commonly reported as a provocative

activity is forward bending and sitting, with the key feature here being the

tendency to hold the lumbar spine into segmental hyper-extension)

Excess of segmental lordosis at symptomatic level with posture and movements

Difficulty assuming and/or maintaining neutral lordotic postures with

a tendency to position themselves into hyper-extension

Pain relief with spinal flexion

Exclusion criteria for nonspecific chronic LBP with motor control

impairment of flexion pattern or active extension pattern

Not fulfilling inclusion criteria: low $(<15)$ Revised Oswestry score,

signs of neurologic involvement (radicular pain), non-mechanical pain,

more generalized pain, evidence of specific diagnosis, e.g., spondylolisthesis,

inflammatory disease, no agreement upon the motor control impairment

between the two independent clinicians

Presence of red flags

Presence of dominant yellow flags

Previous spine surgery, pregnant at the time of the study or 6 months

postpartum, recently undergone a period of motor control rehabilitation

(lumbar multifidus, -7\%; ilio-costalis lumborum pars thoracis, $-6 \%$, and transverse fibers of internal oblique, $-5 \%)$. The flexion relaxation ratio of the back muscles was lower for all nonspecific chronic LBP subjects, suggesting a lack of flexion relaxation of the back muscles including Lumbar Multifidus in all the nonspecific chronic LBP patients.

It was concluded that differences in trunk muscle activity in sitting clearly discriminates the two clinical sub-groups of nonspecific chronic LBP (AEP and FP) from the no-LBP group. The heterogeneity of the nonspecific chronic LBP subjects' muscle activation patterns highlighted the importance of sub-classification and provided further evidence for construct validity to the CS.

These findings also suggest hyperactivity of the Lumbar Multifidus and transverse fibres of internal oblique in the AEP group and the inability to relax these muscles when asked to slump. These novel findings highlighted that excessive co-contraction of stabilising muscles and the inability to relax these muscles was associated with these pain disorders. These findings challenge commonly held beliefs regarding optimal sitting posture and a lack of spine stability as an underlying basis for nonspecific chronic LBP. In contrast, there was a trend of decreased muscle activity in FP patients, accompanied with an increase in lumbar flexion.

This study provides preliminary evidence that should be confirmed in larger sample size studies including magnitudes reporting on the estimates of the discriminative ability of the system.

\section{Can a statistical classification model using laboratory tests (sEMG and lumbo-sacral kinematics) accurately discriminate between two groups of nonspecific chronic LBP patients (AEP and FP) and a group of no-LBP controls? Are the clinically reported sub-group characteristics 'real phenomena'? (Study IV)}

The laboratory tests analysed in Study IV (Dankaerts et al., 2009) involved commonly reported aggravating spinal postures and movements that form part of the basis of the clinical classification process. The results of Study IV demonstrated that a Statistical Classification Model identified five lumbo-sacral kinematics (lower lumbar spine kinematics in sitting and forward bending) and two trunk muscle activation (lack of flexion relaxation of the superficial fibres of lumbar multifidus in slump sitting and end range of forward bending) variables that were able to correctly classify $96.4 \%$ of cases and to discriminate the three groups (no-LBP, AEP and FP). These findings suggest that the sub-group characteristics' reported clinically are 'real phenomena' supporting that the lumbar kinematics of functional postures and movement, and the inability of the superficial lumbar multifidus to relax, were key predictors of nonspecific chronic LBP.

This study provided evidence of construct validity in support of the CS, and highlights the heterogeneity of the nonspecific chronic LBP population with regards to motor control of the lumbar spine and the potential importance of classification in the identification of sub-groups. This finding supports the validity of the clinical process of diagnostics, where a diagnosis is determined as a result of a complete physical evaluation involving a series of tests while closely correlating these findings to the individual's complaints.

7. Can a CS and laboratory tests (trunk muscle activation and lumbo-sacral kinematics) be applied in outcome studies investigating the predictive validity of a CS for a sub-group of nonspecific chronic LBP with a classification of MCI? (Study V)

The results of this case study (Study V) (Dankaerts et al., 2007) of a patient with nonspecific chronic LBP, classified as having a multidirectional MCI (O'Sullivan, 2004, 2005) demonstrated normalised motor control following a targeted motor learning intervention specific to the patient's classification. The improvement in motor control was associated with reductions in pain, disability and fear related to movement. This was obtained at 3 months, and maintained at 6 and 12-months follow-up. Laboratory-based tests supported the classification (pre-intervention) and showed normalisation of motor control at 6 months (including normalisation of flexion relaxation response to forward bending), providing preliminary evidence that the $\mathrm{MCI}$ was the mechanism underlying the LBP disorder.

It was concluded that the use of this CS and laboratory tests (trunk muscle activation and lumbo-sacral kinematics) could be applied in outcome studies investigating the predictive validity of a CS for a sub-group of nonspecific chronic LBP and MCI.

In summary, the five studies conducted provide an accumulation of evidence towards the validation of a proposed mechanismbased CS for nonspecific chronic LBP patients with a classification of $\mathrm{MCl}$. The following section discusses the relevance of these findings with respect to the specific aims of the studies and the background 
literature relevant to the validation of a CS for patients with nonspecific chronic LBP and MCI.

\section{What drives the altered motor control?}

The present series of studies demonstrate that motor control patterns exhibited by patients with AEP and FP are different from each other and from those in healthy subjects. These motor patterns observed in the LBP subjects appear maladaptive and potentially provocative. However there is no clear answer to the question of causality. In other words, it is not known what leads to these very different motor control patterns.

In light of this, it is important to highlight some of the more recent work by our team. Astfalck et al. (2010a,b) showed that similar postural characteristics, but not the EMG patterns, are present in adolescents with nonspecific chronic LBP suggesting that they are present early in development of the disorder. The fact that the EMG changes were not present in these adolescents suggests that the motor changes may evolve with the disorder and may be secondary, whereas the kinematic patterns present early and may in themselves be provocative.

In support of this view a study investigating sagittal standing posture by Smith et al. (2008) in 14 year olds highlighted that some postural sub-groups carry an increased risk for LBP. It was suggested that some of these postural changes may be influenced by developmental and/or genetic factors, or as a response to patterns of loading such as increased BMI, and carry a greater risk for LBP.

Longitudinal prospective studies are necessary to further unravel the question of causality, but to date none have been published. Several recent extensive literature reviews have discussed models explaining the mechanism underlying pain and dysfunction in the low back (for example (Hodges and Moseley, 2003; van Dieen et al., 2003; Moseley and Hodges, 2004). A detailed review of all the potential reasons that underlie these findings is beyond the scope of this review paper, rather the purpose of this section is to discuss the importance of these studies in light of some of the proposed mechanisms.

\section{Why would patients develop behaviours of self provocation?}

While it is not yet fully understood, it is hypothesised that complex changes in the central nervous system result in altered patterns of sensorimotor integration (Moseley, 2003). As a consequence, the chronic LBP patient is left with disrupted feedback sense and altered body schema. This hypothesis fits with findings of impaired repositioning sense (O'Sullivan et al., 2003), impaired two point discrimination (Moseley, 2008; Luomajoki and Moseley, 2009), and cortical changes in chronic LBP patients (Wand et al., 2011). Other factors such as stress, anxiety, patients beliefs and clinicians advise to hold erect postures and co-contract the spine stabilising muscles may also be reflective in these findings. Some of these factors may explain why LBP patients lack awareness of their pain behaviours and display a lack of reflex withdrawal from pain provocative postures and movements.

\section{Motor pattern driven by pain, disability?}

The fact that there was minimal difference in pain and disability levels between the clinical sub-groups, suggests that the motor patterns in the AEP and FP groups was not directly driven by pain experience. This is in contrast with the pain-spasm-pain model, first formally proposed by Travell et al. (1942) suggesting that pain leads to muscle hyperactivity. The results from the above summarized series of studies do not support that the amount of muscle activity is a linear response to the level of reported pain or disability, although a lack of flexion relaxation of the superficial lumbar multifidus was a common feature of both sub-groups.

\section{Motor pattern driven by fear avoidance behaviours?}

It has been suggested that the impact of pain on motor control is secondary to the substantial attention demand inherent to pain caused by psycho-social factors (e.g. beliefs relating to pain, stress, fear of pain and re-injury, depression, hypervigilance, and catastrophising). Indeed, the nature of chronic spinal pain (Burton et al., 1995; Burton and Erg, 1997; Schade et al., 1999) has the ability to disrupt spinal motor control by virtue of its demand on the central nervous system (Watson et al., 1997; Marsh and Geel, 2000; van Dieen et al., 2003; Moseley et al., 2004). There is now substantial evidence for the involvement of areas (such as the pre-motor cortex) that are normally linked to motor function (Casey, 1999) in pain perception. This central integration of nociceptive input in chronic LBP may exert a strong influence on the motor function. It has been suggested that this information is integrated with that from (pre-) frontal areas concerned with future implications of the pain and with establishing response priorities (Price, 2000).

Investigating the correlation between pain-related fear on muscle activity during all sitting trials in Study III demonstrated that there was no significant correlation between the levels of muscle activation of the back muscles (superficial fibres of lumbar multifidus and iliocostalis lumborum pars thoracic) across all back pain patients and pain-related fear. While we acknowledge that this may be the case with other nonspecific chronic LBP subjects with high fear levels, these findings do not support the view that pain-related fear (Vlaeyen and Crombez, 1999) directly modulates the motor control in these sub-groups of nonspecific chronic LBP patients during sitting.

\section{Maladaptive motor control pattern}

While these models may provide some explanation of motor behaviour observed in the LBP population, the data from the series of studies suggest that for the sub-group of nonspecific chronic LBP with a classification of $\mathrm{MCl}$, the motor control strategy was not one that could be regarded as (i) 'efficient' to escape pain, (ii) driven by the direct experience of pain and disability or (iii) driven by levels of fear avoidance beliefs. Studies II-IV demonstrated that the patients consistently postured themselves in the direction of reported pain provocation.

These results support the concept that these patients present with an inherent underlying maladaptive movement behaviour that acts as a potential ongoing peripheral nociceptive drive rather than as a strategy to avoid pain. This kind of provocative pain phenomena has been clearly reported by Sullivan et al. (2009) who reported that repeated lifting tasks resulted in a summation of pain for those with nonspecific chronic LBP. It is our hypothesis that one of the mechanisms for this pain summation is the presence of maladaptive movement behaviours, previously described by Szeto et al. (2005) in neck and arm pain subjects during a typing task.

It is further hypothesised that a multitude of factors could conceivably change the spinal motor control leading to this $\mathrm{MCI}$ such as, physical factors (mechanism of injury, previous injury, where initial adaptive behaviours to pain in the acute phase of a disorder remain and become maladaptive in the chronic phase of the disorder), altered cortical organisation resulting in decreased body awareness and proprioception, cognitive factors such as beliefs about body schema, psychological factors (personality trait) and changes in neuroplasticity secondary to a chronic pain disorder and possible (gender related) genetic factors as well as factors such as the beliefs and training methods of 
physiotherapists that have tended to reinforce training of muscles such as lumbar multifidus.

\section{Clinical implications}

The findings of this series of studies confirm the presence of distinct patterns of $\mathrm{MCI}$ in the AEP and the FP groups. These results advance the clinical understanding of nonspecific chronic LBP patients and have potential implications for the classification and therapeutic management of nonspecific chronic LBP subjects.

Contemporary literature advocates a process of diagnostics, where factors across multiple domains that are involved in these disorders are identified within a bio-psycho-social framework. This is as a result of a complete subjective and physical evaluation that identified the patient's experience of pain, its behaviour, functional impairments, beliefs, coping, pain-related fear and distress. The examination involves analysis of the functional impairments reported by the patient to determine if the movement and pain behaviours are adaptive (protective) or maladaptive (provocative) (Elvey and O'Sullivan, 2004; O'Sullivan, 2004, 2005).

Consistent with this body of clinical literature, Study IV demonstrated that not one single factor but rather a series of factors (involving static posture, dynamic movement including both kinematics and an inability to relax spinal muscles) discriminated between the three groups examined in this study. An important clinical implication of Study IV is therefore that to clinically subclassify nonspecific chronic LBP patients with MCI, clinicians should not simply rely on one test, but rather consistency across a series of functional tests that are linked to the patient's pain behaviour and functional impairments.

Study I demonstrated that clinicians agree on the classification of nonspecific chronic LBP and MCI using this reasoning process. While the above described inter-examiner reliability study suggests that substantial specialised training for clinicians is required, the feasibility to implement the CS into clinical practice seems satisfactory.

The presentation of these patients was commonly related to increased co-contraction of the trunk stabilising muscles during pain provocative tasks, and an inability to relax muscles such as Superficial Lumbar Multifidus during forward bending with associated differences in spinal posture and movement patterning. These findings coupled with other recent research highlighting that absence of timing delays in the transverse abdominis in nonspecific chronic LBP subjects (Gubler et al., 2010), question current clinical beliefs regarding spinal instability and deficits in the spines stabilising muscles as an underlying basis for these disorders. It may in fact be that the increased motor activity of the spines stabilising muscles in some cases results in increased and abnormal loading forces across pain sensitive structures. This may be a reason why clinical trials that target specific training of spinal stabilising muscles for nonspecific chronic LBP are no more effective than other interventions which have small effect sizes (e.g. spinal manipulative therapy) (Ferreira et al., 2006). It was recently demonstrated (Costa et al., 2009) in a randomised placebocontrolled trial that for people with chronic LBP motor control exercise produced short-term improvements in global impression of recovery and activity, but not pain. This also highlights that generic approaches to managing nonspecific chronic LBP using stabilising training do not address the individual motor control deficits identified in patients with the disorder. Acknowledging this factor requires a change in the belief systems of physiotherapists and other health professionals, with a focus less on muscle and more on behaviours and control of movement.

When developing treatment and management for chronic LBP patients it would appear essential to have an understanding of the mechanism underlying the disorder. A behavioural intervention that focuses on cognitive and behavioural change (Cognitive Functional Therapy, CFT) (O'Sullivan, 2004) directed to changing the maladaptive patterns of control and associated cognitive behaviours seems a logical clinical approach when considering this data. This approach involves changing patients beliefs, confronting their fears, providing them awareness of pain mechanisms, enhancing mindfulness of the control of their body during pain provocative functional tasks, training them to reduce excessive trunk muscle activity and change behaviours related to pain provocative postures and movements. This approach has the possibility to reduce the central wind up of pain and disability and unload sensitised spinal structures peripherally (O'Sullivan, 2004). The capacity of this approach to reduce pain, disability and fear as well as normalise abnormal motor control patterns was demonstrated in case Study V (Dankaerts et al., 2007). Preliminary results of recent research investigating the efficacy of CFT in a randomised controlled clinical trial (RCT) supports its efficacy for nonspecific chronic LBP (Fersum et al., 2009b). We await the publication of the full results of this trial.

\section{Limitations of present research}

Despite its' strengths, a number of limitations need to be highlighted. The application of strict inclusion/exclusion criteria (inherent to sub-classification) limits the clinical generalisability of the results of Study II-IV to patients with an AEP and FP classification. The fact no other clinical sub-groups were evaluated was based on logistics and convenience of sampling. It is acknowledged that the nonspecific chronic LBP group consists of other distinct sub-groups than the two sub-groups with clinical signs of $\mathrm{MCI}$.

It is further acknowledged that the results of the EMG studies conducted (Study III and IV) are limited to the superficial muscle sites under examination. Therefore future work should focus on the involvement of deeper muscles deemed important in LBP, such as quadratus lumborum, transversus abdominis, deep lumbar multifidus and psoas (Ingber, 1989; De Franca and Levine, 1991; Hodges and Richardson, 1996; McGill et al., 1996; Andersson et al., 2002; Barker et al., 2004).

Finally, it could be argued that a potential limitation of Study I and IV was the use of 'expert' clinician's classification as 'gold standard' as it is prone to bias. However, in the absence of a true criterion standard for MCI diagnosis, this method reflects current clinical practice (Gracovetsky et al., 1995).

\section{Recommendations for further research}

Further clinical research in the form of RCTs is required, comparing CFT to other 'approaches'. This is the logical and essential next step in the multi-step validation process outlined in this review to further test the predictive validity of the proposed CS.

Further investigation (including brain research) is required into the underlying basis of the development of these patterns and why some patients appear to develop provocative movement behaviours that maintain pain cycles.

\section{References}

Andersson EA, Grundstrom $\mathrm{H}$, Thorstensson A. Diverging intramuscular activity patterns in back and abdominal muscles during trunk rotation. Spine 2002;27 (6):E152-60.

Astfalck R, O'Sullivan P, Straker L, Smith A. A detailed characterisation of pain, disability, physical and psychological features of a small group of adolescents with non-specific chronic low back pain. Manual Therapy 2010a;15(3):240e7.

Astfalck R, O'Sullivan P, Straker L, Smith A, Burnett A, Caneiro J, Dankaerts W. Sitting postures and trunk muscle activity in adolescents with and without nonspecific 
chronic low back pain: an analysis based on subclassification. Spine 2010b;35 (14):1387-95.

Barker K, Shamley D, Jackson D. Changes in the cross-sectional area of multifidus and psoas in patients with unilateral back pain: the relationship to pain and disability. Spine 2004;29(22):E515

Bogduk N, Merskey H. Classification of chronic pain: descriptions of chronic pain syndromes and definitions of pain terms. 2nd ed. International Association for the Study of Pain; 1994.

Burton AK, Erg E. Back injury and work loss. Biomechanical and psychosocial influences. Spine 1997;22(21):2575-80.

Burton AK, Tillotson KM, Main CJ, Hollis S. Psychosacial predictors of outcome in acute and subchronic low back trouble. Spine 1995;20(6):722-8.

Casey KL. Forebrain mechanisms of nociception and pain: analysis through imaging, National Academy of Sciences of the United States of America; 1999:7668-74.

Costa L, Maher C, Latimer J, Hodges P, Herbert R, Refshauge K, et al. Motor control exercise for chronic low back pain: a randomized placebo-controlled trial. Physical Therapy 2009;89(12):1275.

Dankaerts W, O’Sullivan P, Burnett A, Straker L. Altered patterns of superficial trunk muscle activation during sitting in nonspecific chronic low back pain patients: importance of subclassification. Spine 2006a;31(17):2017-23.

Dankaerts W, O'Sullivan P, Burnett A, Straker L. The use of a mechanism-based classification system to evaluate and direct management of a patient with nonspecific chronic low back pain and motor control impairment - a case report. Manual Therapy 2007;12(2):181-91.

Dankaerts W, O'Sullivan P, Burnett A, Straker L, Davey P, Gupta R. Discriminating healthy controls and two clinical subgroups of nonspecific chronic low back pain patients using trunk muscle activation and lumbosacral kinematics of postures and movements: a statistical classification model. Spine 2009;34 (15): 1610 .

Dankaerts W, O'Sullivan PB, Burnett A, Straker LM. Differences in sitting postures are associated with non-specific chronic low back pain disorders when subclassified. Spine 2006b;31(6):698-704.

Dankaerts W, O'Sullivan PB, Straker LM, Burnett AF, Skouen JS. The inter-examiner reliability of a classification method for non-specific chronic low back pain patients with motor control impairment. Manual Therapy 2006c;11(1):28-39.

De Franca G, Levine L. The quadratus lumborum and low back pain. Journal of Manipulative and Physiological Therapeutics 1991;14(2):142.

Elvey R, O'Sullivan P. A contemporary approach to manual therapy. In: Boyling J, Jull G, editors. Grieve's modern manual therapy: the vertebral column. Elsevier; 2004. p. 471-94.

Ferreira P, Ferreira M, Maher C, Herbert R, Refshauge K. Specific stabilisation exercise for spinal and pelvic pain: a systematic review. Australian Journal of Physiotherapy 2006;52:79-98.

Fersum K, O'Sullivan P, Kvåle A, Skouen J. Inter-examiner reliability of a classification system for patients with non-specific low back pain. Manual Therapy 2009a;14:555-61.

Fersum K, O'Sullivan P, Skouen J, Smith A, Kvåle A. Efficacy of classification based 'cognitive functional therapy' in patients with Non Specific Chronic Low Back Pain (NSCLBP) - a randomized controlled trial. 2009b, Sydney.

Ford J. Classification of low back pain (doctoral thesis), Faculty of Medicine, Dentistry and Health Science, The University of Melbourne, Melbourne; 2002.

Fritz J, Delitto A, Erhard R. Comparison of classification-based physical therapy with therapy based on clinical practice guidelines for patients with acute low back pain: a randomized clinical trial. Spine 2003;28(13):1363-72.

Gracovetsky S, Newman N, Pawlowsky M, Lanzo V, Davey B, Robinson L. A database for estimating normal spinal motion derived from noninvasive measurements. Spine 1995;20(9):1036-46.

Gubler D, Mannion AF, Schenk P, Gorelick M, Helbling D, Gerber H, et al. Ultrasound tissue Doppler imaging reveals no delay in abdominal muscle feed-forward activity during rapid arm movements in patients with chronic low back pain. Spine 2010;35(16):1506-13.

Hodges PW, Moseley GL. Pain and motor control of the lumbopelvic region: effect and possible mechanisms. Journal of Electromyography and Kinesiology 2003;13(4):361-70.

Hodges PW, Richardson CA. Inefficient muscular stabilization of the lumbar spine associated with low back pain. A motor control evaluation of transversus abdominis. Spine 1996;21(22):2640-50.
Ingber R. Iliopsoas myofascial dysfunction: a treatable cause of" failed" low back syndrome. Archives of Physical Medicine and Rehabilitation 1989;70 (5):382.

Latremoliere A, Woolf CJ. Central sensitization: a generator of pain hypersensitivity by central neural plasticity. The Journal of Pain 2009;10(9):895-926.

Luomajoki H, Moseley G. Tactile acuity and lumbopelvic motor control in patients with back pain and healthy controls. British Medical Journal; 2009.

Marsh AP, Geel SE. The effect of age on the attentional demands of postural control. Gait Posture 2000;12(2):105-13.

McCarthy CJ, Arnall F, Strimpakos N, Freemont A, Oldham JA. The biopsychosocial classification of non-specific low back pain: a systematic review. Physical Therapy Reviews 2004;9:17-30.

McGill S, Juker D, Kropf P. Appropriately placed surface EMG electrodes reflect deep muscle activity (psoas, quadratus lumborum, abdominal wall) in the lumbar spine. Journal of Biomechanics 1996;29(11):1503-7.

Moseley G. A pain neuromatrix approach to patients with chronic pain. Manual Therapy 2003;8(3):130-40.

Moseley GL. I can't find it! Distorted body image and tactile dysfunction in patients with chronic back pain. Pain 2008;140(1):239-43.

Moseley GL, Hodges P. Chronic pain and motor control. In: Boyling JD, Jull G, editors. Grieve's modern manual therapy. Edingburgh: Churchill Livingstone/Elsevier; 2004.

Moseley GL, Nicholas MK, Hodges PW. Does anticipation of back pain predispose to back trouble? Brain 2004;127(Pt 10):2339-47.

O'Sullivan P. Clinical instability of the lumbar spine: its pathological basis, diagnosis and conservative management. In: Boyling J, Jull G, editors. Grieve's modern manual therapy: the vertebral column. Churchill Livingstone, Elsevier; 2004. p. $311-32$.

O'Sullivan P. Diagnosis and classification of chronic low back pain disorders: maladaptive movement and motor control impairments as underlying mechanism. Manual Therapy 2005;10(4):242-55.

O’Sullivan P, Burnett A, Floyd A, Gadsdon K, Logiudice J, Miller D, et al. Lumbar repositioning deficit in a specific low back pain population. Spine 2003; 28:1074-9.

Petersen T, Thorsen C, Mannische C, Ekdahl C. Classification of non-specific low back pain: a review of the literature on classifications systems relevant to physiotherapy. Physical Therapy Review 1999;4:265-81.

Price DD. Psychological and neural mechanisms of the affective dimension of pain. Science 2000;288(5472):1769-72.

Riddle DL. Classification and low back pain: a review of the literature and critical analysis of selected systems. Physical Therapy 1998;78(7):708-37.

Schade V, Semmer N, Main CJ, Hora J, Boos N. The impact of clinical, morphological, psychosocial and work-related factors on the outcome of lumbar discectomy Pain 1999;80(1-2):239-49.

Smith A, O'Sullivan P, Straker L. Classification of sagittal thoraco-lumbo-pelvic alignment of the adolescent spine in standing and its relationship to low back pain. Spine 2008;33(19):2101-7.

Sullivan M, Thibault P, Andrikonyte J, Butler H, Catchlove R, Larivière C. Psychological influences on repetition-induced summation of activity-related pain in patients with chronic low back pain. Pain 2009;141(1-2):70-8.

Szeto G, Straker L, O'Sullivan P. A comparison of symptomatic and asymptomatic office workers performing monotonous keyboard work-1: neck and shoulder muscle recruitment patterns. Manual Therapy 2005;10(4):270-80.

Travell J, Rinzter S, Herman M. Pain and disability of the shoulder and arm. Journal of the American Medical Association 1942;120:417-22.

van Dieen JH, Cholewicki J, Radebold A. Trunk muscle recruitment patterns in patients with low back pain enhance the stability of the lumbar spine. Spine 2003;28(8):834-41.

Vlaeyen JW, Crombez G. Fear of movement/(re)injury, avoidance and pain disability in chronic low back pain patients. Manual Therapy 1999;4(4):187-95.

Waddell G. The back pain revolution. 2nd ed. Edinburgh: Churchill Livingstone; 2004.

Wand B, Parkitny L, O'Connell N, Luomajoki L, McAuley J, Thacker M, Moseley GL. Cortical changes in chronic lowback pain: Current state of the art and implications for clinical practice. Manual Therapy 2011;16(1):9-14.

Watson PJ, Booker CK, Main CJ. Evidence for the role of psychological factors in abnormal paraspinal activity in patients with chronic low back pain. Journal of Musculoskeletal Pain 1997;5(4):41-56. 